\title{
Effect of Defects on the Line shape of Electron Paramagnetic Resonance Signals from the Single-Molecule Magnet $\mathrm{Mn}_{12}$ : A Theoretical Study
}

\author{
Kyungwha Park ${ }^{1,2, *}$, N. S. Dalal ${ }^{2, \dagger}$, and P. A. Rikvold ${ }^{1,3, \ddagger}$ \\ ${ }^{1}$ School of Computational Science and Information Technology, Florida State University, \\ Tallahassee, Florida 32306-4120 \\ ${ }^{2}$ Department of Chemistry, Florida State University, Tallahassee, Florida 32306-4390 \\ ${ }^{3}$ Center for Materials Research and Technology and Department of Physics, Florida State \\ University, Tallahassee, Florida 32306-4350
}

(November 10, 2018) 


\begin{abstract}
We herein estimate the effect of lattice defects on the line shape of electron paramagnetic resonance (EPR) signals from a single crystal of the $S=10$ single-molecule magnet $\mathrm{Mn}_{12}$ with the external magnetic field along the crystal $c$ axis. A second-order perturbation treatment of an effective single-spin Hamiltonian indicates that a small, random, static misorientation of the magnetic symmetry axes in a crystalline lattice can lead to asymmetric EPR peaks. Full spectra are simulated by calculating probability-distribution functions for the resonant fields, employing distributions in the tilt angle of the easy axis from the $c$ axis, in the uniaxial anisotropy parameter, and in the $g$ factor. We discuss conditions under which the asymmetry in the EPR spectra becomes prominent. The direction and magnitude of the asymmetry provide information on the specific energy levels involved with the EPR transition, the EPR frequency, and the distribution in the tilt angle.
\end{abstract}

PACS numbers: 75.50.Xx, 76.30.-v, 75.45.+j, 61.72.Hh

Typeset using REVTEX 


\section{INTRODUCTION}

The nanomagnetic compound $\left[\mathrm{Mn}_{12} \mathrm{O}_{12}\left(\mathrm{CH}_{3} \mathrm{COO}\right){ }_{16}\left(\mathrm{H}_{2} \mathrm{O}\right)_{4}\right] \cdot 2 \mathrm{CH}_{3} \mathrm{COOH} \cdot 4 \mathrm{H}_{2} \mathrm{O}$ (abbreviated hereafter as $\left.\mathrm{Mn}_{12}\right),{ }^{1}$ first synthesized by Lis, ${ }^{1}$ consists of a core of twelve Mn ions, linked together by oxygen atoms from the bridging acetate ligands. The $\mathrm{Mn}$ ions form the magnetic core, and are shown schematically in Fig. 1. The Mn ions are of mixed valence: eight of them $\left(\mathrm{Mn}^{3+}\right)$ with four unpaired electrons $(S=2)$ form the outer ring, and are ferromagnetically coupled within themselves, to lead to a total spin of $S_{1}=8 \times 2=16$. The other four ions $\left(\mathrm{Mn}^{4+}\right)$ each with $S=3 / 2$ form the center of the core and are arranged in a Mn-O cubane type structure. These are also ferromagnetically coupled within themselves, with a total spin of $S_{2}=4 \times(3 / 2)=6$. The total spin on a $\mathrm{Mn}_{12}$ molecule has been determined to be 10, which can be understood as arising from a strong antiferromagnetic coupling between the two $S_{1}$ and $S_{2}$ sub-systems..$^{2-4}$ The whole molecule may thus be considered as a $S=10$ particle as far as the ground state is concerned, which is of primary interest in the present work. The acetate ligands are covalently bonded via the oxygens to the Mn ions. There are two acetic acid molecules and four $\mathrm{H}_{2}$ molecules solvated in the lattice. The magnetic moments of the $\mathrm{Mn}_{12}$ molecules are most easily aligned along the crystal $c$ axis, which is thus the easy axis for a perfect crystal (without imperfections/dislocations). There is a zero-field energy barrier of approximately $65 \mathrm{~K}$ against magnetization reversal. ${ }^{5,6}$ The effective distance between different $\mathrm{Mn}_{12}$ molecules is approximately $14 \AA$. . $^{1,13,14}$

Earlier, we have examined the effect of possible defects on the linewidths of electron paramagnetic resonance (EPR) spectra for the single-molecule magnet $\mathrm{Mn}_{12},{ }^{13,14}$ and have compared our calculated results with experiments. ${ }^{13,18}$ The imperfections in large complex molecules such as $\mathrm{Mn}_{12}$ could be of many types, such as dislocations ${ }^{7}$ and the disorder caused by the various orientations of the acetate ligand and of the solvent molecules in a unit cell. ${ }^{8}$ So far the origin of the possible defects has not yet been clarified. In the study, ${ }^{13,14}$ we modeled that the defects could be represented by the distributions in the uniaxial anisotropy parameter $D$ (second-order anisotropy) and the $g$-factor. To calculate 
the EPR linewidths, the intermolecular dipolar interactions and the distributions in $D$ and $g$ were considered. For $\mathrm{Mn}_{12}$ the intermolecular dipolar interactions ( $\sim$ a couple of hundred gauss) are substantially smaller than the the effect of the distributions in $D$ and $g(\sim$ a thousand gauss). The detailed estimates of the dipolar interactions and the effect of the distributions in $D$ and $g$, were given in Refs. ${ }^{13,14}$ as a function of temperature, resonance frequency, and the energy levels relevant to the EPR transition. Our calculated results ${ }^{13,14}$ agree well with experiments. ${ }^{13,18}$

In the present study, we describe our theoretical investigation of the effects of lattice defects on the signal line shape of EPR spectra for $\mathrm{Mn}_{12}$. We propose that the lattice defects may cause a small, static, random misorientation of the magnetic symmetry axes of each molecule in the lattice. Here we examine the full microwave absorption line shape as a function of resonance frequency and the energy levels relevant to the EPR transitions, including Gaussian distributions in the tilt-angle (the angle between the magnetization easy axis and the crystal $c$ axis) as well as the distributions in $D$ and $g$. Then we find that consideration of the Gaussian distribution in the tilt angle leads to a line-shape asymmetry in the spectra. Conversely, it might be possible to learn about the defect structure from this asymmetry.

This study was prompted by three factors. First, $\mathrm{Mn}_{12}$ and its analogs, with their high-spin ground states of $S=10$, exhibit novel properties, such as macroscopic quantum tunneling (MQT), ${ }^{9,10}$ whose origin has not yet been fully understood. A new model of MQT, especially for $\mathrm{Mn}_{12}$, involves a direct role for lattice defects. ${ }^{7}$ Second, $\mathrm{Mn}_{12}$ has recently been proposed as a material for potential use in quantum computation. ${ }^{11}$ For this application, a detailed knowledge of the line shape of the EPR absorption spectrum would be required. The proposed method can be applied to microwave transitions between the various spin levels. Third, many earlier high-field EPR studies of $\mathrm{Mn}_{12}$ and related systems have reported asymmetric line shapes, but the origin of the asymmetry has not been clarified. ${ }^{5,12}$ It was assumed that the asymmetry might be an instrumental artifact, resulting from the existence of standing waves in the waveguides. ${ }^{5,12}$ 
Asymmetric EPR line shapes have been known since the early days of EPR spectroscopy. For example, Bleaney and Rubins considered forbidden hyperfine transitions and noted that EPR line shapes from powder samples, which have a distribution of particle orientations, can be asymmetric. ${ }^{15}$ Although we use a standard spin Hamiltonian [Eq. (1)] and routine perturbation technique, our study is distinct from such earlier studies in the following ways: (a) We consider single-crystal samples, with measurements along a well-defined direction such as the crystal $c$ axis. (b) Our system involves the existence of strong intramolecular spinexchange effects within the $\mathrm{Mn}_{12}$ molecule (which is an order of $100 \mathrm{~K}$ ), ${ }^{?}$ which obliterates the hyperfine structure and the effects of any forbidden transitions. (c) The system and experimental situation are characterized by a dominant uniaxial anisotropy term in the spin Hamiltonian and a strong external magnetic field, and are thus quite applicable to high-field EPR. (d) We examine systematically the asymmetry in the EPR line shape as a function of the energy levels between which the microwave transition occurs, the microwave frequency, and the distribution in the tilt angle. Future experimental tests of our predictions for the EPR line shapes of single-molecule magnets should be fruitful.

The remainder of this paper is organized as follows. In Sec. II the model is described, and in Sec. III the resonant field is calculated as a function of the rotation angle $\theta$ (which is the tilt angle of the molecular easy axis with respect to the crystal $c$ axis), and the direction and the magnitude of the asymmetry in the resonant fields are discussed. In Sec. IV we calculate the probability-distribution functions of the resonant fields, including the distributions in $\theta$, $D$, and $g$, to predict the asymmetry in the spectra. In Sec. $\mathrm{V}$ we present a discussion and our conclusions.

\section{MODEL}

In the presence of defects or impurities in a sample, $\mathrm{Mn}_{12}$ molecules can be displaced or rotated from their normal positions. Consequently, each molecule sees a slightly different crystal field caused by the surrounding molecules, compared to that seen in a perfect crystal. 
We propose that this slightly different crystal field seen by a molecule results in a small, static, random misorientation (rotation) of the magnetic symmetry axes of the molecule in the crystalline lattice. The degree of the misorientation can be quantified as the magnetization easy axis of the molecule is rotated by an angle $\theta$ away from the crystal $c$ axis. The majority of the molecules are assumed to have their easy axes almost aligned with the $c$ axis, so the tilt angle $\theta$ is assumed to have a Gaussian distribution about zero with a small standard deviation. Hereafter $a, b$, and $c$ denote the crystal axes, while $x, y$, and $z$ denote the molecular (magnetic) anisotropy axes of a single molecule. In this study, we consider the case of varying the magnetic field at a fixed EPR frequency (roughly 50 to $500 \mathrm{GHz}$ ) when the external magnetic field is applied along the $c$ axis.

We confine our study to the uniaxial molecular magnet, $\mathrm{Mn}_{12}$, because of its very small transverse anisotropy compared to its uniaxial anisotropy. ${ }^{5,17}$ Additional transverse anisotropy terms or small transverse magnetic fields at $\theta=0$ may also produce asymmetry in the spectra, because of nonlinear relationships between the energy cost of an EPR transition and the sweeping field. For $\mathrm{Mn}_{12}$, the negligibly small transverse anisotropy enables us to concentrate on the asymmetry effect caused by a distribution in $\theta$ only.

Our goal is to investigate how static, random rotations of the magnetic anisotropy axes caused by defects affect the line shapes of EPR spectra as functions of frequency and energy level, and to predict EPR spectra which may be compared with experimental data in near future. For the single crystal of $\mathrm{Mn}_{12}$ examined in Refs. 13, 18, it was found that the effects of $D$-strain and $g$-strain are more significant than dipolar interactions. ${ }^{13,14}$ Therefore we need to combine the effects of $D$-strain and $g$-strain with the effect of static, random rotations of the easy axes in order to obtain realistic spectra. After analytically calculating the resonant field as a function of $\theta, D$, and $g$, we numerically obtain the probability distribution function of the resonant field, assuming Gaussian distributions in $\theta, D$ and $g$. We do not take into account the effects of natural linewidths, of dipolar interactions between molecules, or of temperature on the line shapes.

When an external magnetic field is applied along the $c$ axis, and the magnetic anisotropy 
easy axis of a single molecule (the $z$ axis) is tilted by $\theta$ away from the $c$ axis, the singlespin ground-state Hamiltonian is, to lowest order, in terms of the spin operators along the molecular axes,

$$
\mathcal{H}=-D S_{z}^{2}-g \mu_{B} B \cos \theta S_{z}-g \mu_{B} B \sin \theta\left(\sin \psi S_{x}+\cos \psi S_{y}\right)
$$

where $D=0.55 k_{B},{ }^{5}$ the isotropic $g=1.94,{ }^{5} \mu_{B}$ is the Bohr magneton, and $B$ is the magnitude of the external field. Here, $\psi$ is the third of the standard Euler angles $(\theta, \phi, \psi)$ as defined in Ref. 19. (For the rotation matrix, see Appendix A.) There is no constraint on the magnitude of $\psi$ because it does not affect the energy eigenvalues of the spin Hamiltonian.

For simplicity, we ignore small fourth-order anisotropy terms ${ }^{5,6}$ in the single-spin Hamiltonian. This is justifiable because: (i) these terms make rather small energy contributions to the eigenvalues, and (ii) some experimental data indicate that the magnitude of the transverse fourth-order terms for $\mathrm{Mn}_{12}$ may be too small to be measured via, for example, neutron scattering. ${ }^{21,22}$

We can also write the single-spin Hamiltonian in terms of spin operators along the crystal axes. Both forms of the spin Hamiltonian represent the same system. (See Appendix A.)

\section{RESONANT FIELDS}

To calculate perturbatively, to lowest order, the resonant fields with the spin Hamiltonian (1), we assume that the tilt angle $\theta$ is very small. This assumption is probably valid because a large misalignment between the crystal $c$ axis and the magnetic easy axis of a molecule (the $z$ axis) is not expected for $\mathrm{Mn}_{12}$, since the reported $\mathrm{X}$-ray data ${ }^{23}$ on bond angles and distances are quite precise, with no mention of mosaicity issues. Then taking $V \equiv$ $-g \mu_{B} B \sin \theta\left(\sin \psi S_{x}+\cos \psi S_{y}\right)$ as a small perturbation to the rest of the terms in $\mathcal{H}$, we

obtain the second-order perturbed energy $\mathcal{E}_{M_{s}}^{(2)}$ of the level $M_{s}$, where $M_{s}$ is an eigenvalue of the spin operator $S_{z}$ :

$$
\mathcal{E}_{M_{s}}^{(2)}=\frac{\eta^{2}\left(S-M_{s}\right)\left(S+M_{s}+1\right)}{\mathcal{E}_{M_{s}}^{(0)}-\mathcal{E}_{M_{s}+1}^{(0)}}+\frac{\eta^{2}\left(S+M_{s}\right)\left(S-M_{s}+1\right)}{\mathcal{E}_{M_{s}}^{(0)}-\mathcal{E}_{M_{s}-1}^{(0)}},
$$


where $\eta \equiv\left(g \mu_{B} B \sin \theta\right) / 2$, and $\mathcal{E}_{M_{s}}^{(0)}=-D M_{s}^{2}-g \mu_{B} B \cos \theta M_{s}$ is the unperturbed energy eigenvalue of the level $M_{s}$. With the selection rule that EPR transitions occur between adjacent energy levels only, we have at resonance $h \nu=\mathcal{E}_{M_{s} \pm 1}^{(0)}+\mathcal{E}_{M_{s} \pm 1}^{(2)}-\mathcal{E}_{M_{s}}^{(0)}-\mathcal{E}_{M_{s}}^{(2)}$, where $\nu$ is the frequency of the applied microwave radiation in the EPR experiment. The resonance equation is quartic in $B$, so its solutions are too complicated to provide useful information. To obtain a simple analytic expression for the resonant field, we substitute the unperturbed expression for the resonant field $B_{0}$ [for example, for the transition between the levels $M_{s}$ and $\left.M_{s} \pm 1, B_{0}=\left(\mp h \nu-D\left(2 M_{s} \pm 1\right)\right) / g \mu_{B} \cos \theta\right]$, for $B$ in Eq. (2). Then we obtain the approximate resonant fields as follows:

$$
\begin{aligned}
B_{\mathrm{res}}\left(M_{s} \rightarrow M_{s}-1\right)= & B_{0}\left(M_{s} \rightarrow M_{s}-1\right)-\frac{g \mu_{B} B_{0}^{2} \sin ^{2} \theta X\left(\nu, M_{s}\right)}{4 \cos \theta}, M_{s}=10,9, \ldots \\
B_{\mathrm{res}}\left(M_{s} \rightarrow M_{s}+1\right)= & B_{0}\left(M_{s} \rightarrow M_{s}+1\right)+\frac{g \mu_{B} B_{0}^{2} \sin ^{2} \theta X\left(\nu,-M_{s}\right)}{4 \cos \theta}, M_{s}=-10,-9, \ldots \\
X\left(\nu, M_{s}\right)= & \frac{2\left(S-M_{s}+1\right)\left(S+M_{s}\right)}{h \nu}-\frac{\left(S+M_{s}-1\right)\left(S-M_{s}+2\right)}{h \nu-2 D} \\
& -\frac{\left(S-M_{s}\right)\left(S+M_{s}+1\right)}{h \nu+2 D} .
\end{aligned}
$$

Notice that the sign of the term proportional to $X$ for the transition $M_{s} \rightarrow M_{s}-1$ is opposite to that for $M_{s} \rightarrow M_{s}+1$. The first type of transitions, $M_{s} \rightarrow M_{s}-1$, represent transitions among energy levels in the lower-energy potential well (see Fig. 2). The second type, $M_{s} \rightarrow M_{s}+1$, corresponds to transitions among energy levels in the higher-energy potential well (see Fig. 2). At frequencies approximately lower than $100 \mathrm{GHz}$ both types of transitions are observed, while at higher frequencies only transitions in the lower energy are observed. This is because at high frequencies the EPR excitation energy exceeds the difference in adjacent energy levels relevant to the transitions.

Our approximation [Eqs. (3) and (4)] is valid when the absolute value of the ratio between the second-order and the zero-order terms becomes very small compared to unity: $\left|Y\left(\nu, M_{s}, \theta\right)\right| \equiv\left|g \mu_{B} B_{0} \sin ^{2} \theta X\left(\nu, \mp M_{s}\right) /(4 \cos \theta)\right| \ll 1$ for $M_{s} \rightarrow M_{s} \pm 1$. Figure 3 shows $Y\left(\nu, M_{s}, \theta\right)$ as a function of frequency $\nu$ and $M_{s}$ at $\theta=0.1 \mathrm{rad}\left(\approx 5.7^{\circ}\right)$ for the two types of transitions. For the transitions $M_{s} \rightarrow M_{s}-1$ (we consider the case that the resonant 
field is positive at $\theta=0$, that is, $\left.h \nu-D\left(2 M_{s}-1\right)>0\right)$, the approximation becomes good for all $M_{s}$ in the range of frequencies from $50 \mathrm{GHz}$ to $500 \mathrm{GHz}$, except for higher energy levels than $M_{s}=1$ [Fig. 3(a)]. The approximation is better for higher frequencies and larger $M_{s}$ (lower energy levels). For the transitions $M_{s} \rightarrow M_{s}+1$ (we consider the case that $\left.D\left(2 M_{s}-1\right)-h \nu>0\right)$, the approximation becomes better at higher frequencies or for smaller magnitude of $M_{s}$ (higher energy levels) [see Fig. 3(b)]. As shown in Fig. 4, the resonant fields from this approximation agree well with the exact diagonalization results at $\nu=66.135 \mathrm{GHz}^{24}$

Next we examine the consequences of the nonzero $\theta$ on the resonant fields, Eqs. (3) and (4), as functions of the frequency and the energy level. Gaussian distributions in $D$ and $g$ provide symmetric distributions in the resonant fields. However, a Gaussian distribution in $\theta$ lets the molecules have smaller or larger resonant fields than that for $\theta=0$, in an asymmetric fashion. This yields asymmetric tails in the average line shapes of EPR spectra.

To investigate the asymmetry effect as a function of $\nu$ and $M_{s}$, we define the quantity $A\left(\nu, M_{s}, \theta\right) \equiv B_{\text {res }}(\theta \neq 0)-B_{\text {res }}(\theta=0)$. As shown in Figs. 5(a) and 6(a), for $M_{s} \rightarrow M_{s}-1$, the sign of $A\left(\nu, M_{s}, \theta=5.7^{\circ}\right)$ is positive so the resonant fields become minima at $\theta=0$ for the whole examined range of frequencies and for all energy levels in the lower-energy potential well. Therefore, the spectra will have asymmetric tails in the direction of increasing field. The magnitude of $A\left(\nu, M_{s}, \theta=5.7^{\circ}\right)$ increases with decreasing $M_{s}$ at low frequencies (below about $200 \mathrm{GHz}$ ) [Fig. 5(a)] because of high resonant fields, and decreases weakly with decreasing $M_{s}$ at high frequencies [Fig. 6(a)]. For a particular $M_{s}$, the magnitude of $A\left(\nu, M_{s}, \theta=5.7^{\circ}\right)$ increases with increasing frequency, except for $M_{s}=3 \rightarrow 2, M_{s}=2 \rightarrow 1$, and $M_{s}=1 \rightarrow 0$ [Fig. 6(a)]. For $M_{s}=3 \rightarrow 2$ and $M_{s}=2 \rightarrow 1$, the magnitude increases with frequency for low frequencies, and then starts to decrease at about $100 \mathrm{GHz}$ and 250 $\mathrm{GHz}$, respectively. For $M_{s}=1 \rightarrow 0$ the magnitude decreases monotonically with increasing frequency. As shown in Figs. 5(b) and 6(b), for $M_{s} \rightarrow M_{s}+1$, the sign of $A\left(\nu, M_{s}, \theta=5.7^{\circ}\right)$ is negative so the resonant fields attain their maxima at $\theta=0$ for the whole examined range of frequencies and $M_{s}$ in the higher-energy potential well. Thus, the spectra show 
asymmetric tails in the direction of decreasing field. The magnitude of $A\left(\nu, M_{s}, \theta=5.7^{\circ}\right)$ increases with increasing magnitude of $M_{s}$ [Fig. 5 (b)] and with decreasing frequency [Fig. 6(b)], because the resonant fields increase. It should be recalled that our approximation breaks down at low frequencies and large magnitude of $M_{s}$ [Fig. 3(b)].

Here we discuss some previous works using similar theoretical methods. Bleaney ${ }^{20}$ used the similar spin Hamiltonian to Eq. (1) in a strong field limit to examine the hyperfine structure in paramagnetic salts. Friedman and Low calculated the resonant fields for $\mathrm{Mn}^{2+}$ doped into zinc fluosilicate $\left(\mathrm{ZnSiF}_{6}: 6 \mathrm{H}_{2} \mathrm{O}\right)$ with the applied field oriented at an angle with respect to the crystal axes, assuming that the Zeeman energy is much larger than the zero-field anisotropy energy. ${ }^{25}$ This approximation, which is the same as discussed in Appendix A of the present paper, is a proper assumption for $\mathrm{Mn}^{2+}$ in zinc fluosilicate since that system has a small crystal-field anisotropy. If we apply this assumption to the single-molecule magnet $\mathrm{Mn}_{12}$ however, we find that the resonant field within this approximation is in poor agreement with the exact diagonalization results. (See Fig. 4 and Appendix A.) The second-order corrections overestimate the exact results. For $\mathrm{Mn}_{12}$ at most intermediate frequencies, the Zeeman energy is not very large compared to the zero-field anisotropy, and the appropriate expressions for the resonant fields are our Eqs. (3) and (4).

\section{DISTRIBUTION FUNCTIONS FOR THE RESONANT FIELDS}

Since we obtained the resonant fields as functions of $g, D$, and $\theta$ in Eqs. (3) and (4), we now calculate numerically the probability-distribution functions (pdf) for the resonant fields, using Gaussian distributions in $g, D$, and $\theta$, to predict the experimental EPR spectra. In our current study, the asymmetry in the spectra is concerned rather than the relative intensities of the spectra. The effect of the perturbation $V$ due to the nonzero $\theta$ (and the distribution in $\theta$ ) on the relative intensities will be briefly discussed in Sec. V. The pdf of the resonant field, $F_{B}(B)$, can be calculated as follows.

$$
F_{B}(B)=\int d \theta \int d D f_{B, D, \theta}(B, D, \theta),
$$




$$
f_{B, D, \theta}(B, D, \theta)=f_{g, D, \theta}\left(g^{*}(B), D, \theta\right) /\left|\frac{\partial B}{\partial g}\left(g^{*}(B), D, \theta\right)\right|
$$

where $B$ denotes the resonant field, Eq. (3) or (4), and $g^{*}(B)$ is obtained by solving the resonant field Eq. (3) or (4) for $g .{ }^{26}$ The function $f_{g, D, \theta}\left(g^{*}(B), D, \theta\right)$ is the joint pdf of the three random variables $g, D$, and $\theta$, calculated at $g=g^{*}(B)$. For simplicity, we assume that $g, D$, and $\theta$ are statistically independent, so that the joint pdf factorizes. The double integration in Eq. (6) was performed numerically using Mathematica. ${ }^{27}$ Figure 7 shows the pdfs of the resonant fields for a few transitions at low $(\nu=65 \mathrm{GHz}$ and $66.135 \mathrm{GHz})$ and high $(\nu=200 \mathrm{GHz})$ frequencies. Here we use fixed values of the standard deviations of $D$ and $g,\left(\sigma_{D}=0.02 D\right.$ and $\sigma_{g}=0.008 g$, which are the same values used in Ref. 13) and two different values of the standard deviation of $\theta, \sigma_{\theta} \approx 2.9^{\circ}$ and $5.7^{\circ}$.

As expected from the previous section (see Fig. 6), for $M_{s}=2 \rightarrow 1$ [Figs. 7 (a) and (b) for $\nu=66.135 \mathrm{GHz}$ and $\nu=200 \mathrm{GHz}$, respectively], the long tail of each pdf appears on the right hand side of the maximum of the pdf (the peak field). For both frequencies, the long tails are recognizable even for $\sigma_{\theta} \approx 2.9^{\circ}$, and they become prominent for $\sigma_{\theta} \approx 5.7^{\circ}$. For $M_{s}=3 \rightarrow 2$, at $\nu=66.135 \mathrm{GHz}$, it is hard to visually recognize any asymmetry [Fig. 7(c)] because the asymmetry is much smaller and the symmetric linewidth is larger than for $M_{s}=2 \rightarrow 1$. However, we see a small shift of the peak field towards higher fields as $\sigma_{\theta}$ increases from $2.9^{\circ}$ to $5.7^{\circ}$. As the frequency increases, the asymmetry effect is significantly enhanced [Fig. $7(\mathrm{~d})$ ]. For $M_{s}=-4 \rightarrow-3$, a possible asymmetry is expected at low frequencies only, as seen from Fig. 6(b). The peak field shifts slightly towards lower fields as $\sigma_{\theta}$ increases from $2.9^{\circ}$ to $5.7^{\circ}$ [Fig. $\left.7(\mathrm{e})\right]$. Other than that, it is hard to see any asymmetry in the pdf for the same reasons as for $M_{s}=3 \rightarrow 2$. For $M_{s} \rightarrow M_{s}+1$, as the magnitude of $M_{s}$ increases, the asymmetry effect increases substantially [Compare Fig. 7(e) with 7(f)].

To quantify the asymmetry, we calculate the third central moment, $\left\langle(B-\langle B\rangle)^{3}\right\rangle$, of the resonant-field distribution, and the difference, $\left(\langle B\rangle-B_{\text {peak }}\right)$, between the average field and the peak field, for the transitions shown in Fig. 7 (see Tables I and II). If a pdf is symmetric, its third central moment vanishes, and the peak field should coincide with the 
average field. If a long tail appears on the right (left) hand side of the peak field, then the sign of the third central moment is positive (negative). For $M_{s}=2 \rightarrow 1$ and $M_{s}=3 \rightarrow 2$, the signs of $\left\langle(B-\langle B\rangle)^{3}\right\rangle$ and $\left(\langle B\rangle-B_{\text {peak }}\right)$ are positive, whereas for $M_{s}=-4 \rightarrow-3$ and $M_{s}=-10 \rightarrow-9$ they are negative, in agreement with Fig. 7 .

\section{DISCUSSION AND CONCLUSIONS}

As shown in Figs. 5-7, $\sigma_{\theta} \approx 5.7^{\circ}$ is large enough to observe asymmetric tails in the EPR spectra for some frequencies and energy levels, but small enough to be realistic in experimental samples. We separately discuss the asymmetry in the spectra at a particular value of $\sigma_{\theta}$ for $M_{s} \rightarrow M_{s}-1$ and for $M_{s} \rightarrow M_{s}+1$. For $M_{s} \rightarrow M_{s}-1$ (in the lowerenergy potential well), the asymmetry in the spectra is more pronounced for smaller $M_{s}$ and higher frequencies, until the frequency becomes very high. At very high frequencies (about $500 \mathrm{GHz}$ or higher), the asymmetry in the resonant field increases weakly with increasing $M_{s}$, but the asymmetry in the spectra for large $M_{s}$ would be masked by large symmetric linewidths. For $M_{s} \rightarrow M_{s}+1$ (in the higher well), the asymmetry in the spectra is strong for large magnitude of $M_{s}$ and low frequencies, because of high resonant fields. But for very low frequencies (below about $50 \mathrm{GHz}$ ) a different approximation scheme is needed than Eqs. (3) and (4). Since for high frequencies the EPR excitation energy becomes larger than the difference in adjacent energy levels, the transitions $M_{s} \rightarrow M_{s}+1$ have narrow frequency or energy-level windows, in which asymmetric spectra are observable. Thus intermediate frequencies (between about 50 and $100 \mathrm{GHz}$ ) would be optimum for observation of the two different types of asymmetry in the spectra.

So far we have concentrated on the asymmetry in the EPR line shapes without mentioning intensities of the spectra. Here we briefly discuss the effect of the distribution in the tilt angle $\theta$ on the intensities of the EPR spectra. At fixed values of $D, g$, and $\theta$, the power absorbed between the $M_{s}$ and the $M_{s}-1$ energy level is written as

$$
\frac{d \mathcal{E}}{d t}=\mathcal{E}_{M_{s}}\left[\dot{\rho}_{M_{s}, M_{s}}\right]+\mathcal{E}_{M_{s}-1}\left[\dot{\rho}_{M_{s}-1, M_{s}-1}\right]
$$


where $\rho(t)$ is the density matrix of the spin system, $\rho(t)_{m^{\prime} m}=\left\langle m^{\prime}|\rho(t)| m\right\rangle,\left[\dot{\rho}_{M_{s}, M_{s}}\right]$ is the change with time of the population in the $M_{s}$ level. To obtain $\left[\dot{\rho}_{M_{s}, M_{s}}\right]$, the density matrix equation $^{34}$ of the spin system [Eq. (1)] is used. For transition rates between different energy levels, the spin-phonon coupling Hamiltonian ${ }^{33}$ is used. For more technical details, refer to Ref. 13, 34. Near resonance (angular frequency of the external transverse oscillating magnetic field $\left.B_{x}, \omega \sim\left(\mathcal{E}_{M_{s}}-\mathcal{E}_{M_{s}-1}\right) / \hbar\right)$, the power absorption becomes ${ }^{34}$

$$
\frac{d \mathcal{E}}{d t}=\frac{\left(\mathcal{E}_{M_{s}-1}-\mathcal{E}_{M_{s}}\right)}{\hbar^{2}}\left|\left\langle M_{s}\left|B_{x} S_{x}\right| M_{s}-1\right\rangle\right|^{2} \Delta\left(B_{z}\right)\left(\rho_{M_{s}, M_{s}}-\rho_{M_{s}-1, M_{s}-1}\right),
$$

where $\Delta\left(B_{z}\right)$ is a Lorentzian line-shape function ${ }^{13}$ and $\rho_{M_{s}, M_{s}}$ is the population of the $M_{s}$ level. The average power absorption can be calculated by averaging Eq. (9) over the Gaussian distributions in $D, g$, and $\theta$. The relative intensity of the average power absorption at a fixed resonance frequency is determined by the transition probability, the line-shape function, the population difference (Boltzmann factors), and spreads in $D, g$, and $\theta$. The transition probability changes with the energy levels involved with the EPR transition, but it does not change due to the perturbation $V$ caused by the nonzero $\theta$. The reason is that as far as we are interested in the phenomena near resonance, only perturbation terms which oscillate with the frequency close to the resonance frequency are important to the transition probability. Thus, the interaction, $Q(t)=B_{x} S_{x}\left(e^{i \omega t}+e^{-i \omega t}\right) / 2$, between the spin system and the oscillating transverse magnetic field $B_{x}$ does contribute to the transition probabilities, but not the perturbation terms $V$. Then, the maximum height of the line-shape function $\Delta\left(B_{z}\right)$ is determined by a linewidth due to the finite lifetime of an excited state. The lifetime of an excited state does not change much with the perturbation terms $V$, so that the height is not much affected by the nonzero $\theta$. The populations of the $M_{s}$ and $M_{s}-1$ level are not affected by the nonzero $\theta$, because the populations are determined by the Boltzmann factor and small changes in the energy due to $V$ are not noticeable in the Boltzmann factor. Therefore, we conclude that the relative intensity for an EPR transiton between specific energy levels is not affected by the perturbation $V$, and that the effect of the distribution in $\theta$ on the relative intensity is solely due to the spread in $\theta$. 
To observe asymmetric line shapes in EPR experiments, one needs to optimize the experimental conditions. First, the asymmetry is more prominent for smaller $M_{s}$ for $M_{s} \rightarrow M_{s}-1$ (or larger magnitude of $M_{s}$ for $M_{s} \rightarrow M_{s}+1$ ), but for those transitions the spectral intensity is generally poor at low temperatures because of the small populations in the excited states. Thus the sample temperature must be optimized to achieve a reasonable intensity. Second, one must avoid the level crossings at which levels in the two potential energy wells coincide. ${ }^{28}$ If the EPR transitions happen to occur near level-crossing points, the spectra could pick up a large extra line broadening which could prevent one from observing the small asymmetry. As the frequency increases, adjacent energy levels move farther apart, so it becomes easier to avoid level-crossing points in the EPR transitions. Third, one must avoid asymmetries caused by experimental artifacts, such as the presence of standing waves in the EPR probe. ${ }^{5,12}$ Standing waves can cause severe line-shape distortion due to mixing between a dispersion spectrum and an absorption spectrum. This can be avoided by using the resonance cavity EPR technique. ${ }^{29-32}$ Fourth, the choice of frequency is important. To observe two different types of asymmetry (one in the direction of increasing field and the other in the direction of decreasing field) at a single frequency, the frequency must be lower than about $100 \mathrm{GHz}$.

If the asymmetries are observed in spectra with the optimum experimental conditions, one can estimate the distributions in $D$ and $g$ from the linewidths of almost symmetric spectra. Then from the spectra with significant asymmetries, we can estimate how broadly the easy axes of the molecules are distributed in the sample. The width of the distribution in $\theta$ may provide information on the defect concentration. Additionally the distribution in $\theta$ may partially contribute to the distribution in the tunnel splittings, which was recently proposed $^{7}$ and measured experimentally, ${ }^{35}$ because nonzero $\theta$ produces the transverse terms relevant to tunneling from one potential well to another.

In summary, we have theoretically examined the effect of defects on the line shapes of EPR spectra for field sweeps with the quasi-static external field along the crystal $c$ axis at fixed frequencies in the range of $50 \mathrm{GHz}$ to $500 \mathrm{GHz}$ for a single crystal of the single-molecule 
magnet $\mathrm{Mn}_{12}$. Static, random rotations of the magnetization easy axes of the molecules, due to defects, lead to asymmetries in the spectra. The strength and direction of the asymmetry depend on the frequency, the energy level, and the width of the distribution in the tilt angle $\theta$ (which depends on the defect concentration). With carefully chosen frequencies, energy levels, and the appropriate experimental technique, the asymmetry effect should be observable in future experiments. Comparison of the observed and calculated spectra could yield direct and quantitative information on the distribution in orientations and the concentration of defects in the samples. This information may also be useful to understand the origin of magnetization tunneling in the molecular magnet $\mathrm{Mn}_{12}$. Experimental examination of our predictions should be quite fruitful.

\section{Acknowledgments}

We are grateful to M. A. Novotny and S. Hill for useful discussions. This work was funded by NSF Grant Nos. DMR-9871455, DMR-0120310, and DMR-0103290, and by Florida State University through the School of Computational Science and Information Technology and the Center for Materials Research and Technology.

\section{APPENDIX A}

The spin Hamiltonian shown in this Appendix is equivalent to the spin Hamiltonian (1), but the approximation used in the calculations are different from those used in Sec. III.

To rewrite this spin Hamiltonian in terms of the spin operators along the crystal axes, we use the following rotation matrix, ${ }^{19}$

$\left(\begin{array}{c}S_{x} \\ S_{y} \\ S_{z}\end{array}\right)=\left(\begin{array}{ccc}\cos \psi \cos \phi-\cos \theta \sin \phi \sin \psi & \cos \psi \sin \phi+\cos \theta \cos \phi \sin \psi & \sin \psi \sin \theta \\ -\sin \psi \cos \phi-\cos \theta \sin \phi \cos \psi & -\sin \psi \sin \phi+\cos \theta \cos \phi \cos \psi & \cos \psi \sin \theta \\ \sin \theta \sin \phi & -\sin \theta \cos \phi & \cos \theta\end{array}\right)\left(\begin{array}{c}S_{a} \\ S_{b} \\ S_{c}\end{array}\right)$

where $(\theta, \phi, \psi)$ are the three Euler angles. ${ }^{19}$ Then we obtain 


$$
\begin{aligned}
\mathcal{H}= & -D\left[\sin ^{2} \theta \sin ^{2} \phi S_{a}^{2}+\sin ^{2} \theta \cos ^{2} \phi S_{b}^{2}+\cos ^{2} \theta S_{c}^{2}-\sin ^{2} \theta \sin \phi \cos \phi\left\{S_{a}, S_{b}\right\}\right. \\
& \left.-\sin \theta \cos \theta \cos \phi\left\{S_{b}, S_{c}\right\}+\sin \theta \cos \theta \sin \phi\left\{S_{a}, S_{c}\right\}\right]-g \mu_{B} B S_{c}
\end{aligned}
$$

where $\{A, B\}$ is the anticommutator.

To calculate the resonant fields, we here assume that the Zeeman energy is much larger than the zero-field anisotropy energy, so that the eigenvalues $M_{s}^{\prime}$ of $S_{c}$ are good quantum numbers. Taking the Zeeman energy, $-g \mu_{B} B S_{c}$, as an unperturbed spin Hamiltonian (taking the quantization axis as the direction of the external magnetic field) and the other terms as small perturbations, we obtain the resonant fields as follows:

$$
\begin{aligned}
B_{\mathrm{res}}\left(M_{s}^{\prime} \rightarrow M_{s}^{\prime}-1\right)= & \frac{1}{g \mu_{B}}\left[h \nu-\frac{2 M_{s}^{\prime}-1}{2} D\left(3 \cos ^{2} \theta-1\right)\right. \\
& +\frac{D^{2} \sin ^{2} \theta \cos ^{2} \theta}{2 h \nu}\left(431+24 M_{s}^{\prime}-24 M_{s}^{\prime 2}\right) \\
& \left.+\frac{D^{2} \sin ^{4} \theta}{8 h \nu}\left(-217-6 M_{s}^{\prime}+6 M_{s}^{\prime 2}\right)\right], \\
B_{\mathrm{res}}\left(M_{s}^{\prime} \rightarrow M_{s}^{\prime}+1\right)= & \frac{1}{g \mu_{B}}\left[-h \nu-\frac{2 M_{s}^{\prime}+1}{2} D\left(3 \cos ^{2} \theta-1\right)\right. \\
& +\frac{D^{2} \sin ^{2} \theta \cos ^{2} \theta}{2 h \nu}\left(-431+24 M_{s}^{\prime}+24 M_{s}^{\prime 2}\right) \\
& \left.+\frac{D^{2} \sin ^{4} \theta}{8 h \nu}\left(217-6 M_{s}^{\prime}-6 M_{s}^{\prime 2}\right)\right] .
\end{aligned}
$$

The above results were also shown in Ref. 25. As shown in Fig. 4, the resonant fields in this approximation do not agree well with the exact diagonalization results, in contrast to Eqs. (3) and (4). For most intermediate frequencies, the Zeeman energy is not very large compared to the zero-field anisotropy energy, and the approximation of Eqs. (3) and (4) is therefore to be preferred. 


\section{REFERENCES}

*Electronic address: park@csit.fsu.edu

† Electronic address: dalal@chem.fsu.edu

$\ddagger$ Electronic address: rikvold@csit.fsu.edu

${ }^{1}$ T. Lis, Acta Crystallogr. B 36, 2042 (1980).

${ }^{2}$ R. Sessoli, D. Gatteschi, A. Caneschi, and M. A. Novak, Nature (London) 365, 141 (1993).

${ }^{3}$ R. A. Robinson, P. J. Brown, D. N. Argyriou, D. N. Hendrickson, and S. M. J. Aubin, J. Phys.: Condens. Matter 12, 2805 (2000).

${ }^{4}$ T. Goto, T. Kubo, T. Koshiba, J. Arai, Y. Fujii, A. Oyamada, K. Takeda, and K. Awaga, Physica B 284-288, 1227 (2000).

${ }^{5}$ A. L. Barra, D. Gatteschi, and R. Sessoli, Phys. Rev. B 56, 8192 (1997).

${ }^{6}$ I. Mirebeau, M. Hennion, H. Casalta, H. Andres, H. U. Güdel, A. V. Irodova, and A. Caneschi, Phys. Rev. Lett. 83, 628 (1999).

${ }^{7}$ E. M. Chudnovsky and D. A. Garanin, Phys. Rev. Lett. 87, 187203 (2001); Phys. Rev. B 65, $094423(2002)$.

${ }^{8}$ A. Cornia, R. Sessoli, L. Sorace, D. Gatteschi, A. L. Barra, and C. Daiguebonne, condmat/0112112 (unpublished).

${ }^{9}$ Quantum Tunneling of Magnetization - QTM '94, Vol. 301 of NATO Advanced Study Institute, Series E: Applied Sciences, edited by L. Gunther and B. Barbara (Kluwer, Dordrecht, 1995).

${ }^{10}$ E. M. Chudnovsky and J. Tejada, Macroscopic Quantum Tunneling of the Magnetic Moment, Cambridge Studies in Magnetism, Vol. 4 (Cambridge University Press, Cambridge, 1998) and references therein. 
${ }^{11}$ M. N. Leuenberger and D. Loss, Nature (London) 410, 789 (2001).

${ }^{12}$ A. L. Barra, D. Gatteschi, and R. Sessoli, Chem-Eur. J. 6, 1608 (2000).

${ }^{13}$ K. Park, M. A. Novotny, N. S. Dalal, S. Hill, and P. A. Rikvold, Phys. Rev. B 65, 014426 (2002).

${ }^{14}$ K. Park, M. A. Novotny, N. S. Dalal, S. Hill, and P. A. Rikvold, Phys. Rev. B (in press).

${ }^{15}$ B. Bleaney and R. S. Rubins, Proc. Phys. Soc. (London) 77, 103 (1961); 78, 778 (1961).

${ }^{16}$ B. Bleaney, Phil. Mag. 43, 441 (1951).

${ }^{17}$ J. A. A. J. Perenboom, J. S. Brooks, S. Hill, T. Hathaway, and N. S. Dalal, Phys. Rev. B 58, $330(1998)$.

${ }^{18}$ S. Hill, S. Maccagnano, K. Park, R. M. Achey, J. M. North, and N. S. Dalal, Phys. Rev. B 65, 224410 (2002).

${ }^{19}$ H. Goldstein, Classical Mechanics (Addison-Wesley, Reading, 1980).

${ }^{20}$ B. Bleaney, Phil. Mag. 43, 441 (1951).

${ }^{21}$ W. Bao, R. A. Robinson, J. R. Friedman, H. Casalta, E. Rumberger, and D. N. Hendrickson, cond-mat/0008042 (unpublished).

${ }^{22}$ I. Mirebeau, M. Hennion, H. Casalta, H. Andres, H. U. Güdel, A. V. Irodova, and A. Caneschi, cond-mat/0009233 (unpublished).

${ }^{23}$ X-ray data exp. paper

${ }^{24}$ The frequency $\nu=66.135 \mathrm{GHz}$ corresponds to that used in Ref. 29 .

${ }^{25}$ E. Friedman and W. Low, Phys. Rev. 120, 408 (1960).

${ }^{26}$ A. Papoulis, Probability, Random Variables, and Stochastic Processes (McGraw-Hill, New York, 1965). 
${ }^{27}$ S. Wolfram, The Mathematica Book, 3rd edition (Wolfram Media/Cambridge University Press, New York, 1996).

${ }^{28}$ L. D. Landau, Phys. Z. Sowjetunion 2, 46 (1932); C. Zener, Proc. R. Soc. London, Ser. A 137, 696 (1932).

${ }^{29}$ S. Hill, J. A. A. J. Perenboom, N. S. Dalal, T. Hathaway, T. Stalcup, and J. S. Brooks, Phys. Rev. Lett. 80, 2453 (1998).

${ }^{30}$ E. del Barco, J. M. Hernandez, J. Tejada, N. Biskup, R. Achey, I. Rutel, N. S. Dalal, and J. Brooks, Phys. Rev. B 62, 3018 (2000).

${ }^{31}$ R. Blinc, P. Cevc, D. Arčon, N. S. Dalal, and R. M. Achey, Phys. Rev. B 63, 212401 (2001).

${ }^{32}$ S. Maccagnano, R. Achey, E. Negusse, A. Lussier, M. M. Mola, S. Hill, and N. S. Dalal, Polyhedron 20, 1441 (2001).

${ }^{33}$ M. N. Leuenberger and D. Loss, Phys. Rev. B 61, 1286 (2000).

${ }^{34}$ K. Blum, Density Matrix Theory and Applications, 2nd edition (Plenum, New York, 1996).

${ }^{35}$ K. M. Mertes, Y. Suzuki, M. P. Sarachik, Y. Paltiel, H. Shtrikman, E. Zeldov, E. Rumberger, D. N. Hendrickson, and G. Christou, Phys. Rev. Lett. 87, 227205 (2001). 


\section{TABLES}

TABLE I. Third central moments of the resonant-field distribution, and the difference between the average and peak resonant fields at $\nu=65 \mathrm{GHz}, 66.135 \mathrm{GHz}$, and $200 \mathrm{GHz}$ for $\sigma_{\theta} \approx 2.9^{\circ}$, $\sigma_{D} \approx 0.02 D$, and $\sigma_{g} \approx 0.008 g$ (see the solid curves in Fig. 7). The third root is taken for the third central moments to give the same units for both measures of the asymmetry. For $M_{s}=-4$, the asymmetry is too small to obtain a reliable moment and difference.

\begin{tabular}{rrcc}
\hline \hline frequency $(\mathrm{GHz})$ & energy level $M_{s}$ & $\sqrt[3]{\left\langle(B-\langle B\rangle)^{3}\right\rangle}($ tesla $)$ & $\langle B\rangle-B_{\text {peak }}$ (tesla) \\
\hline 66.135 & 2 & 0.023 & $3.02 \times 10^{-3}$ \\
\hline 66.135 & 3 & 0.0052 & $1.90 \times 10^{-4}$ \\
\hline 65 & -10 & -0.27 & $-6.68 \times 10^{-2}$ \\
\hline 200 & 2 & 0.030 & $3.07 \times 10^{-3}$ \\
\hline 200 & 3 & 0.025 & $2.40 \times 10^{-3}$ \\
\hline \hline
\end{tabular}

TABLE II. Third central moments of the resonant-field distribution, and the difference between the average and peak resonant fields at $\nu=65 \mathrm{GHz}, 66.135 \mathrm{GHz}$, and $200 \mathrm{GHz}$ with the same values of $\sigma_{D}$ and $\sigma_{g}$ as in Table I, but with $\sigma_{\theta}=5.7^{\circ}$ (see dashed curves in Fig. 7).

\begin{tabular}{rrcc}
\hline \hline frequency $(\mathrm{GHz})$ & energy level $M_{s}$ & $\sqrt[3]{\left\langle(B-\langle B\rangle)^{3}\right\rangle}($ tesla $)$ & $\langle B\rangle-B_{\text {peak }}$ (tesla) \\
\hline 66.135 & 2 & 0.068 & $9.89 \times 10^{-3}$ \\
\hline 66.135 & 3 & 0.016 & $1.11 \times 10^{-3}$ \\
\hline 65 & -10 & -0.93 & $-4.46 \times 10^{-1}$ \\
\hline 200 & 2 & 0.098 & $2.69 \times 10^{-2}$ \\
\hline 200 & 3 & 0.091 & $2.13 \times 10^{-2}$ \\
\hline \hline
\end{tabular}




\section{FIGURES}

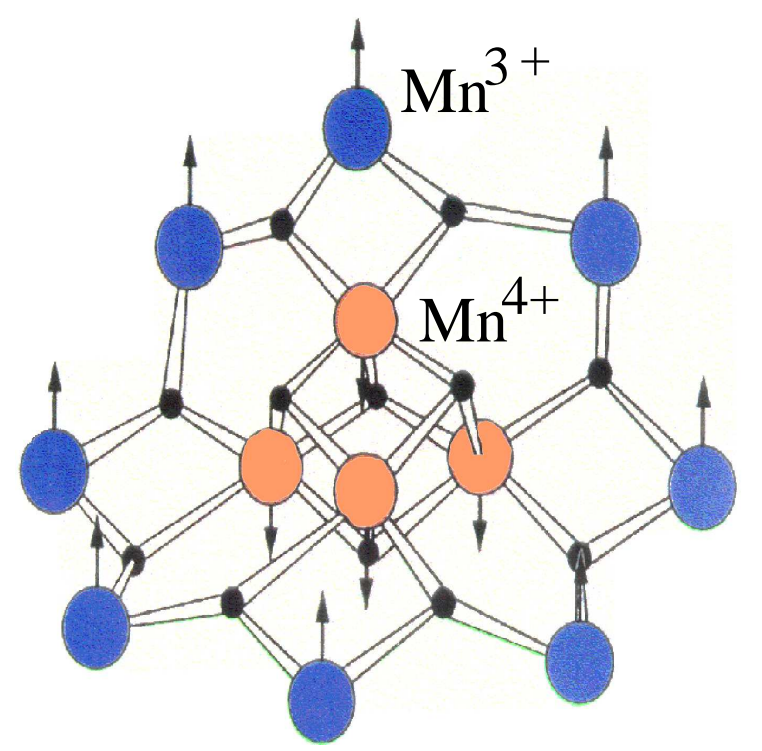

FIG. 1. Schematic diagram of the magnetic core of the $\mathrm{Mn}_{12}$ molecule. Each molecule includes a tetrahedron of four $\mathrm{Mn}^{4+}(S=3 / 2)$ ions at the center, surrounded by eight $\mathrm{Mn}^{3+}(S=2)$ ions, yielding an effective ground-state spin of $S=10(S=8 \times 2-4 \times 3 / 2)$. Each molecule has a tetragonal symmetry.

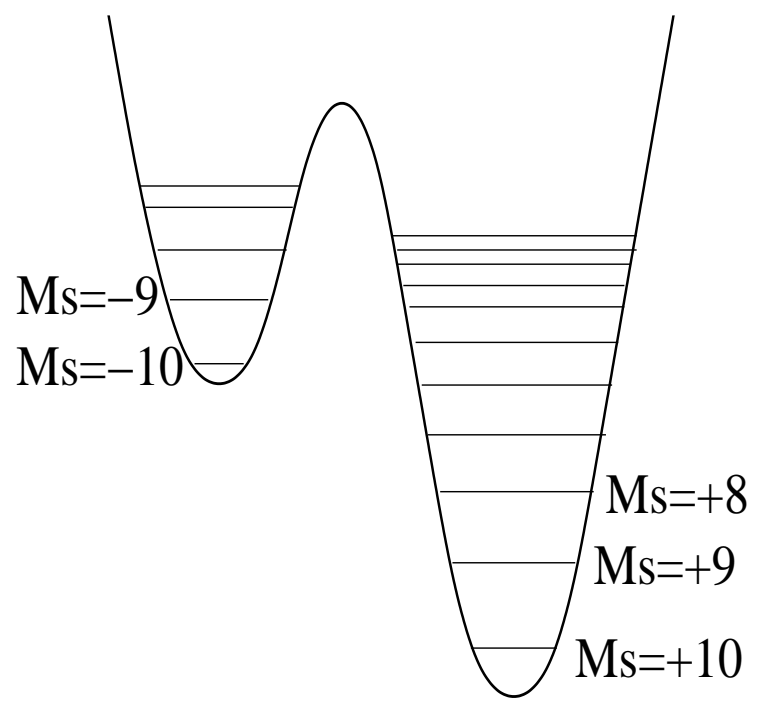


FIG. 2. Schematic diagram of the potential-energy wells for the molecular magnet $\mathrm{Mn}_{12}$, which has an effective spin $S=10$. Here $M_{s}$ is the eigenvalue of the spin operator $S_{z}$. For the lower energy well (the right-hand well), the difference between adjacent energy levels is $g \mu_{B} B+D\left(2 M_{s}-1\right)$. For the higher energy well (the left-hand well), the difference is $-g \mu_{B} B+D\left(2 M_{s}-1\right)$. As the applied field increases, the difference between the energy levels $M_{s}=-10$ and $M_{s}=+10$ increases.
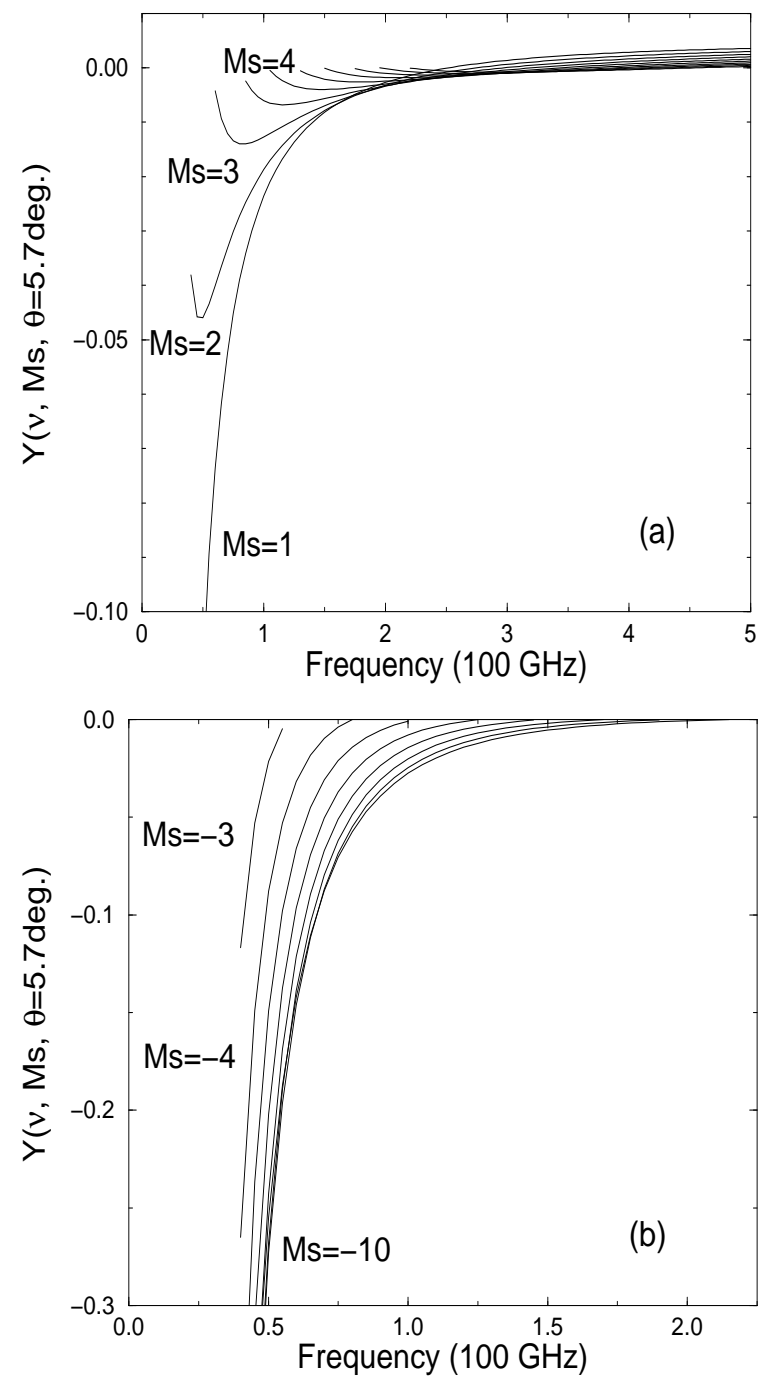

FIG. 3. The ratio between the second-order and the zero-order terms in the resonant fields, $Y\left(\nu, M_{s}, \theta\right)$, vs frequency at $\theta \approx 5.7^{\circ}$ for the transitions (a) $M_{s} \rightarrow M_{s}-1$ and (b) $M_{s} \rightarrow M_{s}+1$. See details in the text. Equations (3) and (4) are valid when $\left|Y\left(\nu, M_{s}, \theta\right)\right| \ll 1$. In (a) $M_{s}=1$ represents the transition $M_{s}=1 \rightarrow 0$. In (b) $M_{s}=-3$ represents the transition $M_{s}=-3 \rightarrow-2$. 

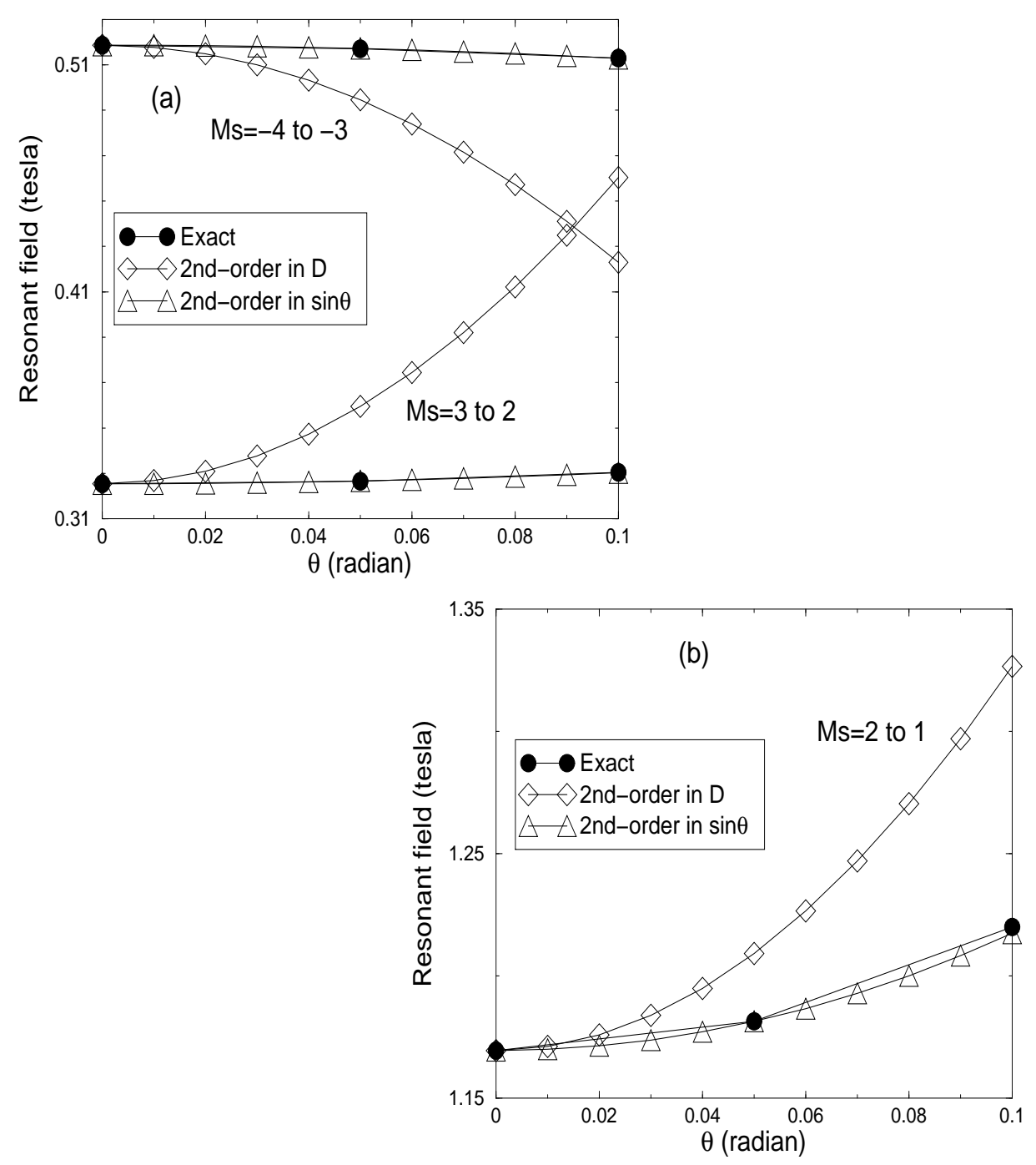

FIG. 4. Resonant fields vs tilt angle $\theta$ at $\nu=66.135 \mathrm{GHz}$ for the transitions (a) $M_{s}=3 \rightarrow 2$ and $M_{s}=-4 \rightarrow-3$, and (b) $M_{s}=2 \rightarrow 1$. The filled circles denote the exact results from numerical diagonalization of the spin Hamiltonian. The triangles denote the results from Eqs. (3) and (4). The diamonds denote the results from Eqs. (A2) and (A3). Eqs. (3) and (4) show good agreement with the exact results, in contrast to Eqs. (A2) and (A3). 

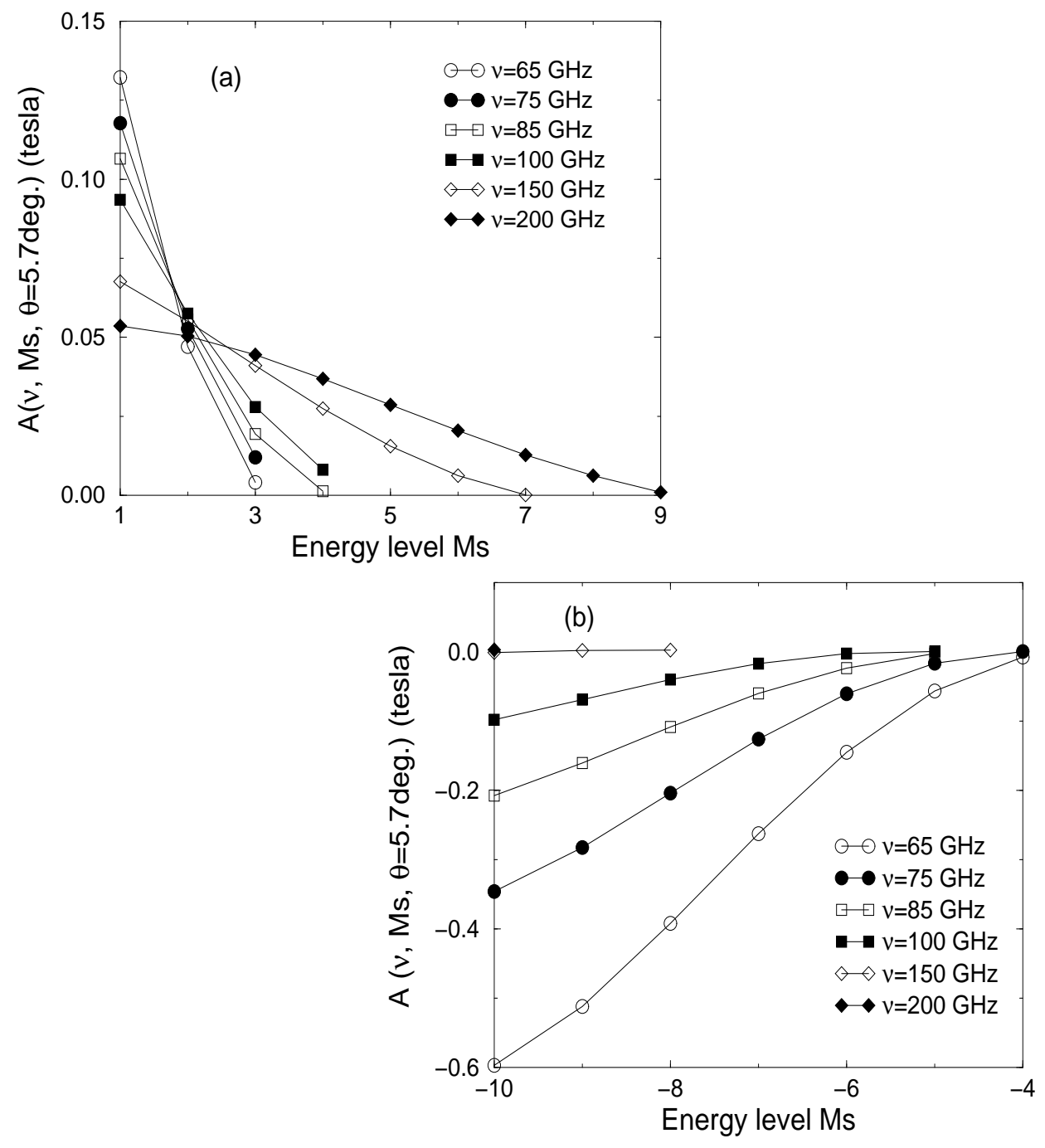

FIG. 5. Asymmetry $A\left(\nu, M_{s}, \theta=5.7^{\circ}\right)$ vs $M_{s}$ at various frequencies for the transitions (a) $M_{s} \rightarrow M_{s}-1$ and (b) $M_{s} \rightarrow M_{s}+1$. See details in the text.

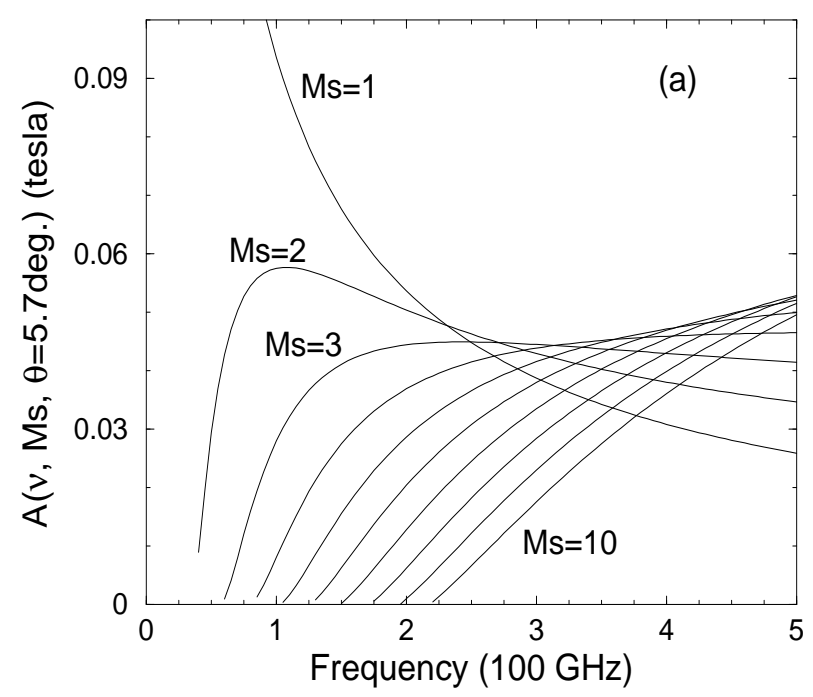




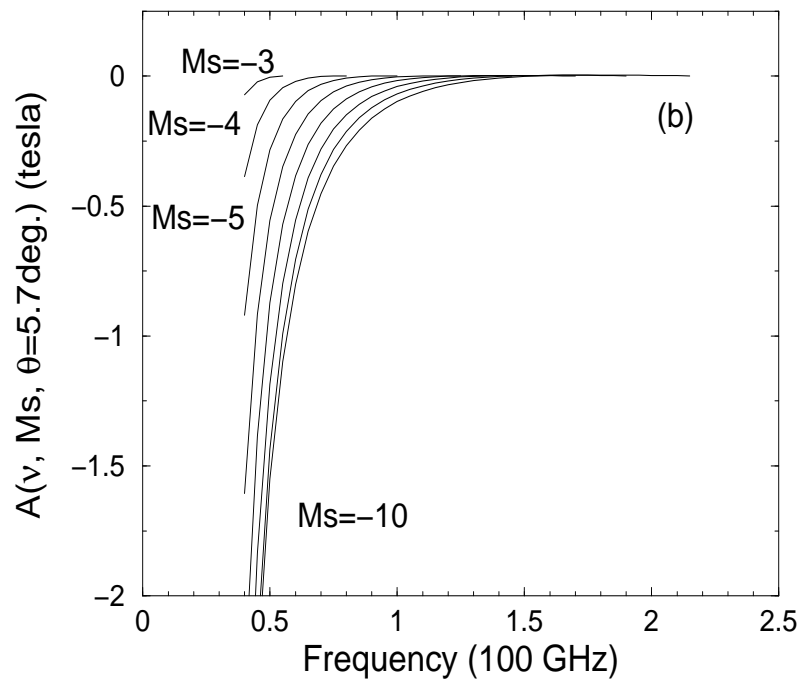

FIG. 6. Asymmetry $A\left(\nu, M_{s}, \theta=5.7^{\circ}\right)$ vs the frequency $\nu$ for the transitions (a) $M_{s} \rightarrow M_{s}-1$ and (b) $M_{s} \rightarrow M_{s}+1$. See details in the text.
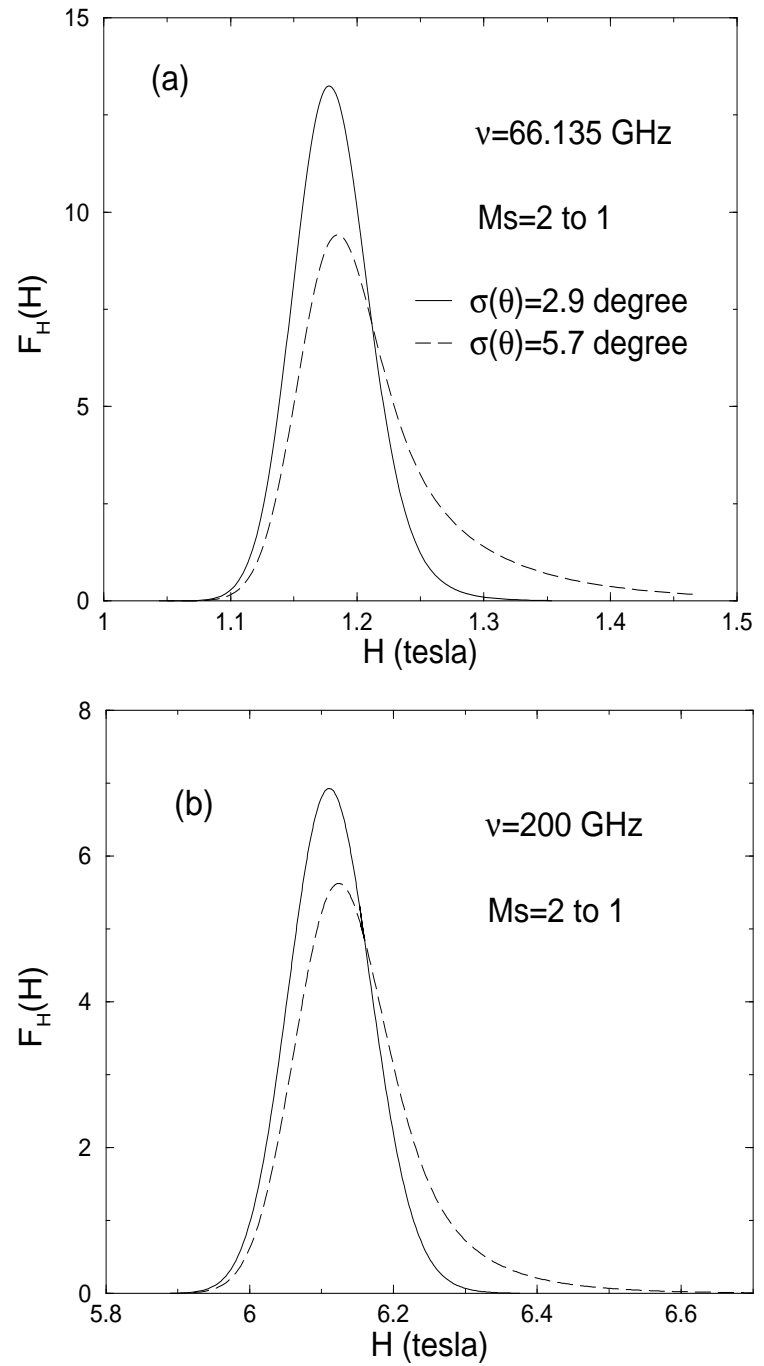

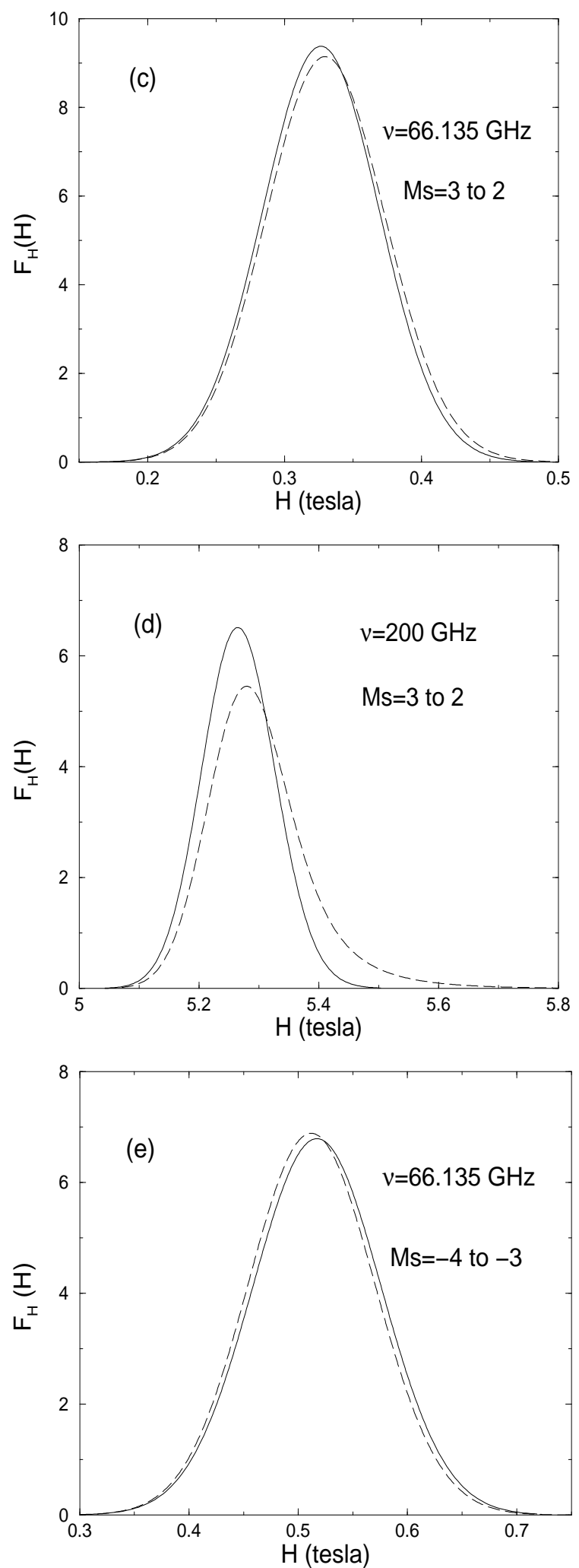


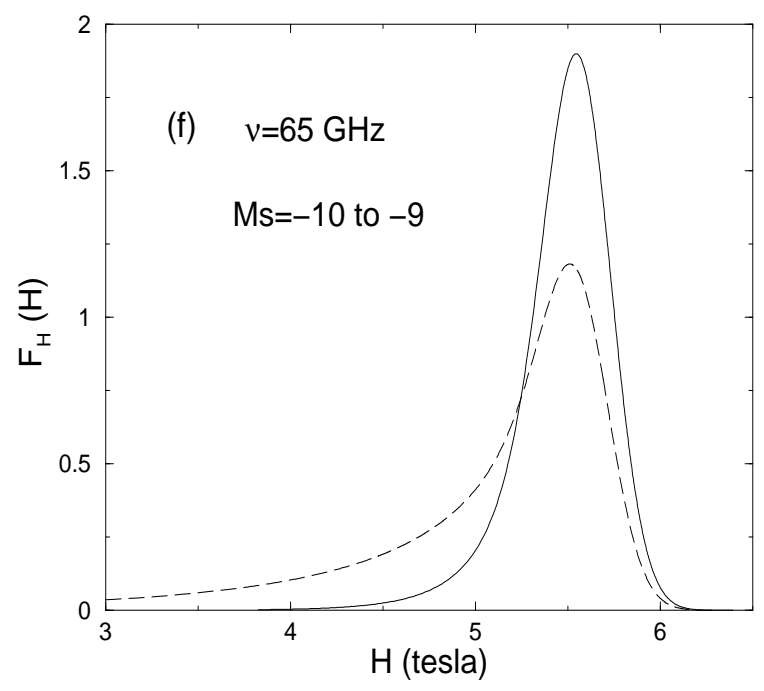

FIG. 7. The probability distribution functions for the resonant fields for the transitions: (a) $M_{s}=2 \rightarrow 1,\left(\right.$ c) $M_{s}=3 \rightarrow 2,\left(\right.$ e) $M_{s}=-4 \rightarrow-3$ at $\nu=66.135 \mathrm{GHz},(\mathrm{b}) M_{s}=2 \rightarrow 1$, (d) $M_{s}=3 \rightarrow 2$ at $\nu=200 \mathrm{GHz}$, and (f) $M_{s}=-10 \rightarrow-9$ at $\nu=65 \mathrm{GHz}$, when $\vec{B} \| \hat{c}$. Here we use the standard deviations of $D$ as $0.02 D$, the standard deviation of $g$ as $0.008 g$, and the standard devation of $\theta$ as $2.9^{\circ}$ (solid curves) and $5.7^{\circ}$ (dashed curves). 